\title{
The effect of additional off-diagonal disorder of interionic interaction on charge-storage in sub-nanometer pores of supramolecular carbon supercapacitors
}

\author{
Klapchuk M., Grygorchak I. \\ Lviv Polytechnic National University, \\ 12 S. Bandera Str., Lviv, 79013, Ukraine
}

(Received 26 October 2018)

\begin{abstract}
In this work, we report sub-nanoporous carbon which is synthesized for the first time by means of supramolecular engineering methods for efficient energy storage at the electrode/electrolyte interface. The achieved value of the differential capacitance more than twice exceeds the corresponding value for the known carbon structures. Based on the constructed impedance model and microscopic theory, the physical mechanisms of the investigated processes are suggested.
\end{abstract}

Keywords: supramolecular assemblies, nanoporous carbon, porous structure, impedance spectroscopy, Nyquist diagram, 1D model of EDL, equation-of-motion method.

2000 MSC: 82D15, 82D20

UDC: 541.136 .2

DOI: $10.23939 / \mathrm{mmc} 2018.02 .147$

\section{Introduction}

The rapid development of electric vehicles and alternative energy, telecommunication and computer technology actualizes the task of radically improving of the operating parameters of existing devices for the electrical energy storage and the creation of new ones - the so-called supercapacitors, whose capacitance is provided by the electrical double layer (EDL) on the boundary of the porous solid phase and electrolyte. However, despite the considerable efforts, the successes achieved in this way cannot yet be regarded as spectacular: the differential capacitance value $C_{\text {diff }}$ for known classes of activated carbon structures and electrolyte systems does not exceed $15 \div 17 \mu \mathrm{F} \mathrm{cm}^{-2}$ [1]. This value is far from the highest value $\left(\sim 50 \mu \mathrm{F} \mathrm{cm}^{-2}[2]\right)$ inherent to the surface of liquid mercury, in which the length of the Thomas-Fermi screening is significantly less than that of nanoporous carbonates. It is gratifying that the analysis of the latest references (see for example, [3-7]) finally shows the correct trend in relevant studies: the transition from the unsystematic involvement of more and more new materials (which in structure, price and etc. is more likely to be "amusing", without any reasoned broad-based chance of taking into account tough market demands regarding the price of one Farad and environmental safety) towards deeper understanding of the physical mechanisms of energy and charge storage in the EDL. It becomes clear that trying to succeed in a given perspective makes no sense without finding answers to a number of the following important issues:

- the algorithm of conjugation of the optimal porous structure of carbon with its corresponding electronic structure, which would ensure the release of a Helmholtz capacitance by the capacitance of the space charge region in a solid;

- interconnection of the structure of the electron energy spectrum with the decrease of the repulsive interaction of surface-sorbed ions;

- limitation of the differential capacitance by various factors, in particular quantum capacitance and the Coulomb blockade of EDL formation;

- the influence of the fractal geometry of nanoporous carbon on the kinetics of electric current and on the rapid diffusion of ions in sub-nanometer pores. 
The latter problem is well focused in the work [8]. The authors found a significant increase in the capacitance of carbon pores of diameter $(d)$, less than $1 \mathrm{~nm}$ : with decreasing $d$ from $1.5 \mathrm{~nm}$ to $0.8 \mathrm{~nm}$, the differential capacitance increases from 4 to $14 \mu \mathrm{F} \mathrm{cm}^{-2}$. Despite the fact that the achieved value of $C_{\text {diff }}$ is not something extraordinary, and the nature of such growth, as well as the mechanism of diffusion of ions in this case are not clearly understood, these results can be extremely useful for constructing theoretical mechanisms of energy storage in nanoporous environments, giving a powerful tool for technologists and experimenters to radically improve the performance of supercapacitors. This paper highlights the importance of both theoretical and experimental contribution to solve this problem.

\section{Basic concepts and methods of experiment}

The stated goal was achieved using in the processes of energy storage the third basic type of the organization of substances, i. e. clathrate (or supramolecular) due to its structural and energy peculiarities $[9,10]$. This can lead not only to fundamentally new regularities of interphase charge transfer, but also to highly efficient capacitive energy storage. Therefore, taking into account the needs of ecological safety and low cost, $\beta$-cyclodextrin was chosen as a precursor for activation carbonization; its structure is schematically depicted in Fig. 1.

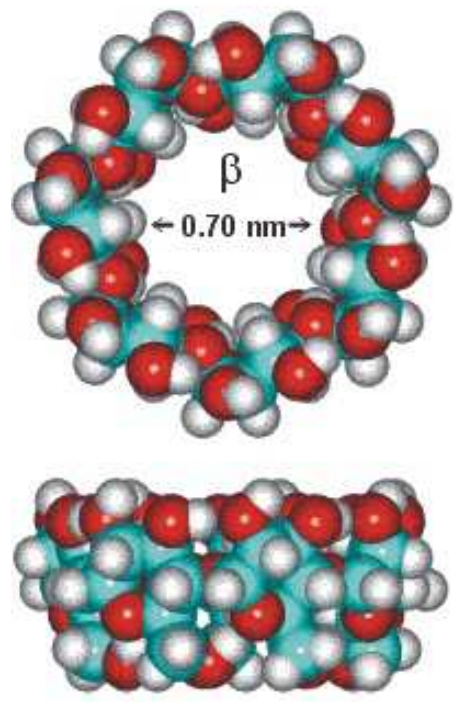

Fig. 1. Image of $\beta$-cyclodextrin molecules [11].

It contains intramolecular cavities, in which guest components can be accommodated by molecular recognition based on the principle of "lockkey" [10]. Moreover, due to their self-organization the cyclodextrins can form so-called molecular beads with an internal hollow channel of subnanometer sizes, which can be changed by different modes of pyrolysis. The formation of subnanoporous carbon was carried out under the following regimes: heating of the initial precursor at a rate of $1^{\circ} \mathrm{C} / \mathrm{min}$ to $100^{\circ} \mathrm{C}$ and endurance for water removal for 2 hours, stabilization at a temperature of $500{ }^{\circ} \mathrm{C}$ for 5 hours, carbonization at $800^{\circ} \mathrm{C}$ for 4 hours. X-ray spectral analysis showed almost pure amorphous carbon phase of the obtained product.

The porous structure of the carbon obtained was determined by precision porosimetry methods using a porometer ASAP $2000 \mathrm{M}$.

Electrochemical measurements in two- and three-electrode circuits with a silver chloride electrode comparison were carried out. As an electrolyte, $30 \%$ aqueous $\mathrm{KOH}$ solution was used. Electrode potentials $E$ are listed in relation to the standard hydrogen electrode comparison.

Impedance spectra (in the frequency range $5 \times 10^{-3}-10^{5} \mathrm{~Hz}$ with amplitude of $5 \mathrm{mV}$ ) in potentiostatic conditions and galvanostatic charge - discharge cycles were recorded using the measuring complex "AUTOLAB" of the company "ECO CHEMIE" (the Netherlands), complete with computer programs FRA-2 and GPES. Deletion of questionable points was done by the Dirichlet filter [11,12]. Frequency dependences of complex impedance $Z$ were analyzed by graphical and analytical method in the software package environment ZView 2.3 (Scribner Associates). Errors of approximation did not exceed $4 \%$. The adequacy of the built impedance models of the experimental data package was confirmed by the completely random nature of the frequency dependencies of the residual first-order differences $[11,12]$. The values of the capacitances for the construction of the capacitance-voltage dependencies were determined with an accuracy of $2-8 \%$ (Kramers-Kronig test was within $10^{-6}-10^{-5}$ ). 


\section{Results and discussion}

\subsection{Experimental results}

The data of precision measurements, elaborated by DFT method, indicate the bimodal porous structure of the synthesized carbon with maxima in pore distribution at their diameters less than $1 \mathrm{~nm}$ and at $4 \mathrm{~nm}$ (Fig. 2).

In a symmetric configuration of a supercapacitor's cell, the specific capacitance reaches $158 \mathrm{~F} / \mathrm{g}$, and in the negative potential region $203 \mathrm{~F} / \mathrm{g}$ with practically "ideal" galvanostatic "charge-discharge" cycles, the Coulomb efficiency of which is $\sim 99.8 \%$ (insert to Fig. 2). Taking into account the values of the area of the active surface, we calculate the differential capacitance, which was $\sim 22 \mu \mathrm{F} \mathrm{cm}{ }^{-2}$, which is higher than the corresponding parameter of known types of activated carbon. A further increase of the $C_{\text {diff }}$ up to $38 \mu \mathrm{F} \mathrm{cm}^{-2}$ was achieved due to an increase in the density of states at the Fermi level by doping the original precursor with a ferrum.

Nyquist diagram of synthesized cavitate carbon is shown in Fig. 3. It certifies the capacitive mechanism of energy storage at the electrode-electrolyte boundary, i. e., the absence of diffusion control inherent in the pseudocapacitors. At the same time, the presence of high-frequency arcs on the hodograph of the impedance (indicated by an arrow) is usually treated by the presence of cylindrical pores closed on one side [12]. The constructed Nyquist diagram corresponds to the de Levy circuit diagram, which was modified according to the Woitte approach by sequentially joining the parallel $R_{S C} C_{S C}$ link (insert to Fig. 3). Here $R_{S C}$ and $C_{S C}$ are resistance and capacitance of the spatial charge area, respectively. It is obvious that the kinetics of capacitive charge storage improves (in particular, the tangent of the angle of electrical losses decreases) both in the case of the growth of the density of states at the Fermi level, and in the case of imposing a negative (in relation to equilibrium) potential. Taking into account that the specific capacitance increases in the indicated potential region, we can purposefully "construct" active supercapacitor systems by attracting so-called "positive" carbon.

Whereas the experimental results are extraordinarily, namely, the colossal growth of the differential capacitance in comparison with known carbons, the improvement of the kinetics of capacitive charge storage, their correlation with the density of states at the Fermi level, then appears the need for their theoretical explanation. We need to develop the theory of investigated processes that will promote their deeper understanding, and hence the further increase in the efficiency of energy storage at the

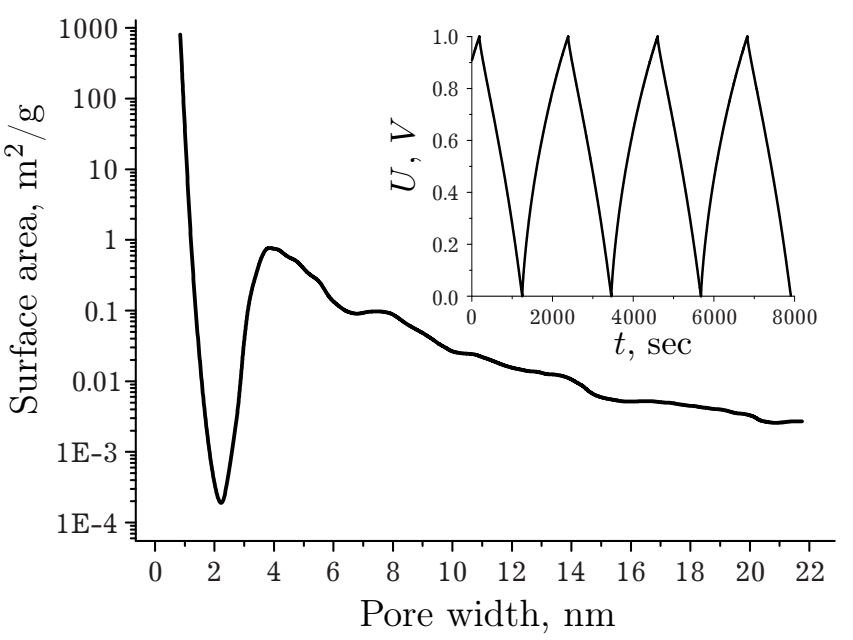

Fig. 2. Distribution of pore space by their $\beta$-cyclodextrin carbon diameter. On the insert is galvanostatic cycles "charge-discharge".

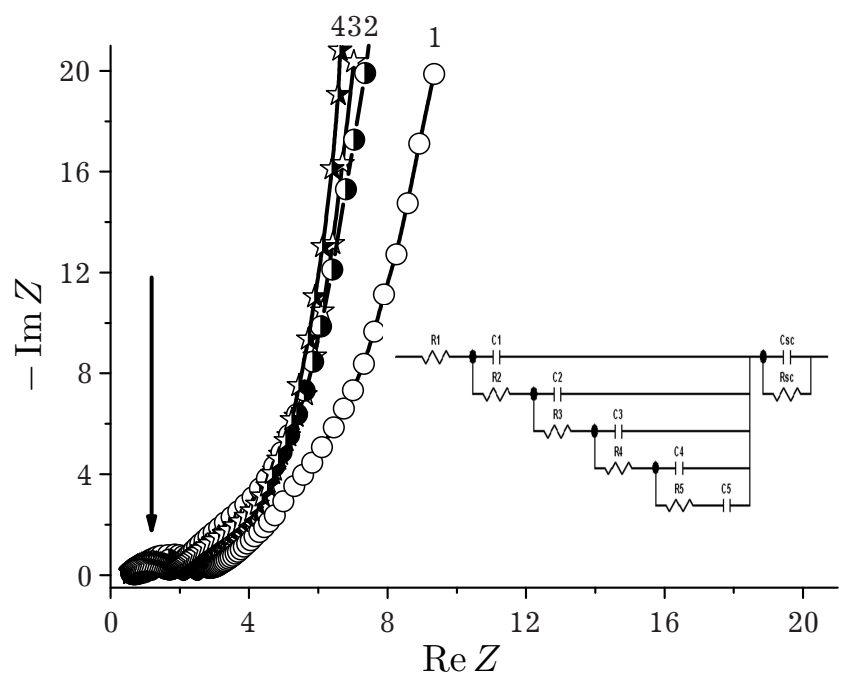

Fig. 3. Nyquist diagrams for $\beta$-cyclodextrin carbon in the case of non doping $(1,2)$ and doping with a ferrum $(3,4)$ at equilibrium potential $(1,3)$ and with negative polarization $(2,4)$. On the insert is a forward electrical circuit. 
boundary of nanoporous carbon with electrolyte. Obviously, first of all, it is necessary to understand the mechanisms of possible release of Helmholtz capacitance by the capacitance of the spatial space charge and/or to reduce the repulsive interaction of superficially sorbed ions in subnanopores and how this will be affected by diffusion processes in spaces with nano-bound geometry. Proceeding from the stated tasks of constructing a microscopic theory, we were based on the following principles.

\subsection{Theoretical approaches}

Previous theoretical approaches have focused on the concept of superionic state that has been suggested in [13], and further explored and generalized in statistical mechanics theories [14] and computer simulations [15] to explain high capacitance in sub-nanometer pores. Using a matrix operator formalism and 1D lattice model, including ion correlation screened by ion-image interaction analytical expression were obtained for the accumulated charge and capacitance as a function of voltage [16]. Cation and anions of comparable size were lined up the single chain (along the axis of the cylindrical narrow pore). One-dimensional model of the EDL was generalized in the framework of statistical-mechanical theory [17] based on the Ising model, and the differential capacitance and energy per unit area of the pore surface were obtained. The graphic dependencies of the calculated capacitance and the average spin as a function of voltage show the fact that the smaller the ions charging the pore, the higher the voltages at which the peaks of the capacitance appear. The peak of capacitance is higher when the ions that charge pore are smaller.

To elucidate the mechanism of charge storage in sub-nanometer pores we use an exactly solvable 1D lattice model where the interaction between ions take place via nearest neighbor hopping. We calculate the density of the electronic states of the system with impurities in low concentration. Furthermore, we investigate the effect of the additional off-diagonal disorder of the interionic interaction on the electronic spectrum, and analyze their significance for the processes of charge storage in such systems. We study the localization effects in the considerable case of disordered 1D system where disorder is applied in form of randomly distributed realistic ions, leading to partly periodic Hamiltonian matrices.

\subsection{Hamiltonian of the system}

In the representation of the secondary quantization, the Hamiltonian of the electronic system in the chain (does not involve the spin variables) can be written as:

$$
H=\sum_{i j} V\left(\mathbf{r}_{i}-\mathbf{r}_{j}\right) a_{i}^{+} a_{j}+E \sum_{i} a_{i}^{+} a_{i}
$$

where $a_{i}^{+}$and $a_{i}$ are the fermion creation and annihilation operators at site $\mathbf{r}_{i}$ for the electronic system. Also $E$ is an onsite energy which do not depend on site number and the interaction $V$ is a function of radius-vector difference on cell $i$ and $j$. Specifically, we start with the equation-of-motion (EOM) method in standard form:

$$
\left[\xi_{k}, H\right]=\varepsilon_{k} \xi_{k}
$$

Let us introduce the operators $\xi_{k}, \xi_{k}^{+}$in the following way $\xi_{k}=\sum_{i} u_{i k} a_{i}, \xi_{k^{\prime}}^{+}=\sum_{j} u_{j k^{\prime}}^{*} a_{j}^{+}$in order to satisfy the residual relations $\left\{\xi_{k}, \xi_{k^{\prime}}^{+}\right\}=\delta_{k k^{\prime}}$ and normalization condition $\sum_{j} u_{j k} u_{j k^{\prime}}^{*}=\delta_{k k^{\prime}}$. Using (2), it leads to

$$
\sum_{i} u_{i k}\left\{\sum_{j} V\left(r_{i}-r_{j}\right) a_{j}+\sum_{j} E a_{j} \delta_{i j}\right\}=\varepsilon_{k} \sum_{j} u_{j k} a_{j} .
$$

Mathematical Modeling and Computing, Vol.5, No. 2, pp. 147-157 (2018) 
By equating the coefficients for $a_{j}$, we get the following relationship:

$$
\sum_{i}\left(V\left(r_{i}-r_{j}\right)+\left(E-\varepsilon_{k}\right) \delta_{i j}\right) u_{i k}=0 .
$$

The energy spectrum of the system can be calculated from equation

$$
\operatorname{det}\left|V\left(r_{i}-r_{j}\right)+\left(E-\varepsilon_{k}\right) \delta_{i j}\right|=0 .
$$

We come to calculate $N \times N$ dimensional secular problem. This approach needs a computationally expensive inversion, whose complexity scales with the second power of the number of involved ions. However, the recursive algorithm can combine accuracy and relatively low numerical cost.

\subsection{Recursive algorithm. Case of nearest neighbors}

We set the matrix elements $V$ in (3) in this way: $V_{i, i \pm 1}=V, V_{i i}=E_{0}$, the rest of them are $V_{i j}=0$, which correspond to the case of nearest neighbors. The probability of a transition of an electron through a site is assumed to be zero. In other words, the wave function of the electron is strongly localized on the site and does not overlap with the wave function of the next neighbors. Thus, it is sufficient to employ a matrix representation of (3):

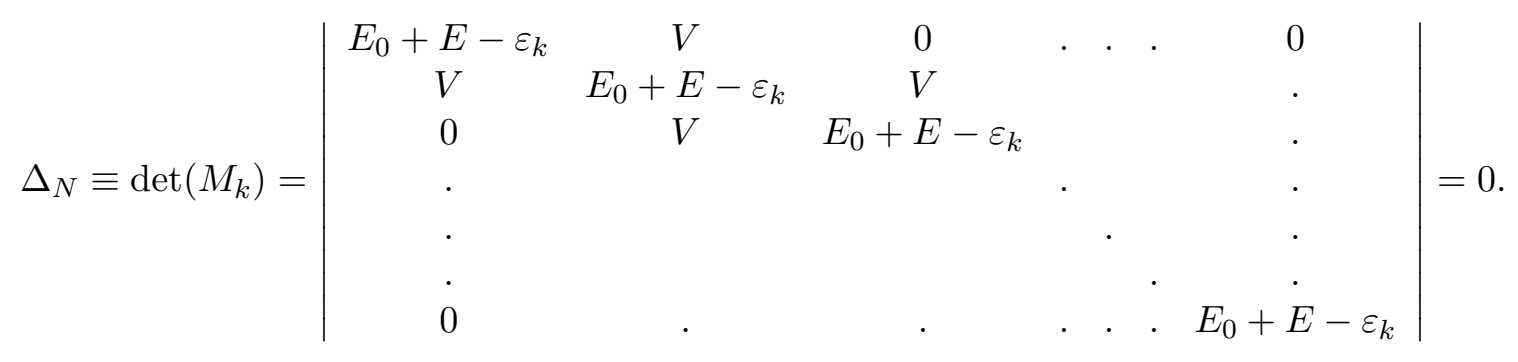

A tridiagonal matrix technique for $\operatorname{det}\left(M_{k}\right)$ calculation have been considered in the general case in the work [18]. Let us introduce new variable $x=\left(\varepsilon_{k}-E-E_{0}\right) / V$ and rewrite determinant $\Delta_{N}$ as follows:

$$
\Delta_{N}=\left|\begin{array}{ccccccc}
-x & 1 & 0 & \cdot & \cdot & \cdot & 0 \\
1 & -x & 1 & & & & \cdot \\
0 & 1 & -x & & & \cdot \\
\cdot & & & \cdot & & & \cdot \\
\cdot & & & & \cdot & 0 \\
\cdot & & & & & 0 \\
0 & . & . & . & 0 & 1 & -x
\end{array}\right|=0
$$

or the corresponding expression can be deduced from above in the recurrent form:

$$
\Delta_{N}=-x \Delta_{N-1}-\Delta_{N-2} .
$$

Given recurrent formula originates the Chebyshev polynomials

$$
\Delta_{0}=1, \quad \Delta_{1}=-x, \quad \Delta_{2}=x^{2}-1, \quad \Delta_{3}=-x^{3}+2 x, \ldots
$$

For $x=-2 \cos \Theta$, we obtain

$$
\Delta_{N}=\frac{\sin [(N+1) \Theta]}{\sin \Theta} .
$$

The condition $\Delta_{N}=0$ gives $\Theta=\frac{k \pi}{N+1}(k=1,2, \ldots, N)$, thus, $x=-2 \cos \frac{k \pi}{N+1}$. Finally, for energy levels in an explicit form we find: $\varepsilon_{k}=E_{0}+E-2 V \cos \frac{k \pi}{N+1}$. 


\subsection{Calculation of the energy spectrum for a local impurity}

Let us consider 1D case (let's have $N$ sites). The electronic properties of the system can be modified by the doped atoms. The impurity is characterized by new matrix elements of the Hamiltonian, which differ from the values inherent to the ideal case, only in the impurity's confinement. Let the impurity site have energy $E_{1}$, the rest of them are chosen to be equal $E_{i>0}=E_{0}$ and interaction $V$ is the same around impurity site. We can consider both the non-diagonal disorder of matrix elements $V_{l l^{\prime}}$ and the diagonal disorder of energy levels $\varepsilon_{l}$.

Such a disorder can be due to two reasons:

- there may be a physical or chemical difference between components of periodically placed sites;

- possible fluctuations in the relative distance between the atomic centers in the chain, and the disorder is obtained as a result of these fluctuations.

Thus, the Hamiltonian has the form

$$
H=V \sum_{i=1}^{N-1}\left(a_{i}^{+} a_{i+1}+a_{i}^{+} a_{i-1}\right)+\sum_{i \neq 0} E_{0} a_{i}^{+} a_{i}+E_{1} a_{0}^{+} a_{0} .
$$

Analogically for the case of the nearest neighbors we obtain the determinant

$$
\Delta_{N}=\left|\begin{array}{ccccccc}
E_{0}-\varepsilon & V & 0 & . & . & . & 0 \\
V & E_{0}-\varepsilon & V & . & . & . & 0 \\
. & & . & & & & . \\
. & & & . & & & . \\
. & & & & . & & . \\
0 & . & . & . & V & E_{0}-\varepsilon & V \\
0 & . & . & . & . & V & E_{1}-\varepsilon
\end{array}\right| .
$$

Using dimensionless values $x=\frac{\varepsilon-E_{0}}{V}, x_{1}=\frac{\varepsilon-E_{1}}{V}$ and expanding $\Delta_{N}$ by its last row, we obtain two additions, the latter one we expand by its last column. Finally, we get the determinant as follows

$$
\Delta_{N}=\left(-x_{1}\right) \Delta_{N-1}^{0}-\Delta_{N-2}^{0},
$$

where

$$
\Delta_{N}^{0}=\left|\begin{array}{cccccc}
-x & 1 & 0 & . & . & 0 \\
1 & -x & 1 & & & \cdot \\
0 & & \cdot & & & \cdot \\
\cdot & & & \cdot & & 0 \\
\cdot & & & 1 & -x & 1 \\
0 & . & . & . & 1 & -x
\end{array}\right|=\frac{\sin [(N+1) \Theta]}{\sin \Theta} .
$$

Thus, we get the equation of the order $N$ :

$$
\Delta_{N}=\left(-x_{1}\right) \sin (N \arccos (-x / 2))-\sin [(N-1) \arccos (-x / 2)]=0 .
$$

By introducing the disturbance $\Delta \equiv\left(E_{0}-E_{1}\right) / V$ and rewriting $x_{1}=\left(\varepsilon-E_{1}\right) / V=x+\Delta$, we have

$$
\Delta_{N}=\sin ((N+1) \arccos (-x / 2)-\Delta \sin (N \arccos (-x / 2))=0 .
$$

In representation of $\Theta$ variables we obtain a periodical function $f(\Theta)=\sin ((N+1) \Theta)-\Delta \sin (N \Theta)=0$ with $N$ roots at $|x| \leqslant 2$ taking into account the condition $x=-2 \cos \Theta$.

To get solutions at $|x| \geqslant 2$ we need to make the transition to complex variables $\Theta \rightarrow i \alpha$. Then from equation $\sin ((N+1) \alpha)-\Delta \sin (N \alpha)=0$ we have $(N-1)$ roots, and impurity induces one decoupled level whose position depends on the impurity strength. The condition for the emergence of such a level is following: $\Delta>(N+1) / N$. Since the impurity changes the energy structure of the porous carbon, it has a great influence on the charge storage in the system. 


\subsection{Effect of additional off-diagonal disorder}

It is interesting to consider the energy spectrum in the case when the potential of the impurity $V_{1}$, that is, the matrix elements $V_{i j}$ differ in the nearest neighbors of the defect, i. e. to study the influence of additional impurity potential on the energy spectrum. In this case, the Hamiltonian has the following structure

$$
H=V \sum_{i=1}^{N-1}\left(a_{i}^{+} a_{i+1}+a_{i}^{+} a_{i-1}\right)+V_{1} a_{0}^{+} a_{1}+\sum_{i \neq 0} E_{0} a_{i}^{+} a_{i}+E_{1} a_{0}^{+} a_{0}
$$

and the determinant becomes

$$
\Delta_{N}=\left|\begin{array}{ccccc}
E_{1}-\varepsilon & V_{1} & & & 0 \\
V_{1} & E_{0}-\varepsilon & V & & \\
& V & E_{0}-\varepsilon & V & \\
& & & V & E_{0}-\varepsilon
\end{array}\right|
$$

Using notations $\left(E_{1}-\varepsilon\right) / V=-x_{1}, x_{1}=x-\Delta$, and defining $V_{1} / V=c / b$ as a parameter relating to the modification of the hopping integral around impurity site, we obtain the equation

$$
\begin{aligned}
\Delta_{N}=\sin (N+1) \Theta & +\Delta \sin N \Theta \\
& +\left(1-\left(V_{1} / V\right)^{2}\right) \sin (N-1) \Theta .
\end{aligned}
$$

We now show some numerical results to examine the role of onsite energy. In Fig. 4 , the energy plots in the special case where $V_{1} / V=4$, compared to the latter, as discussed earlier. A nonzero value for $V_{1}$ leads to a shift either to the left (when $V_{1}>0$ ) or to the right (when $\left.V_{1}<0\right)$.

In the case of the repulsion potential the inclusion of the additional ratio $V_{1} / V$ leads to stronger electronic binding to the impurity when the impurity hopping parameter is large. In other words, it leads to a greater

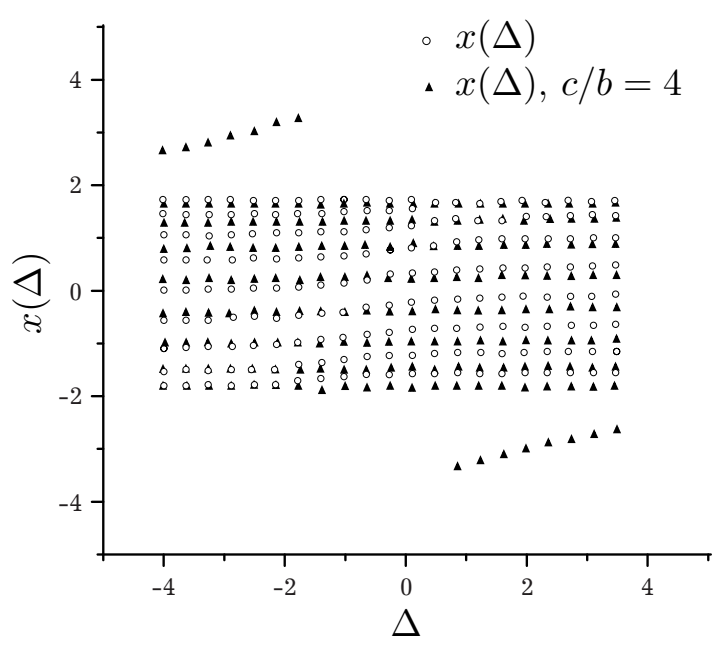

Fig. 4. The effect of additional hopping parameter at energy spectrum, taking $V_{1} / V=4$. decoupling of impurity level.

\subsection{Calculation of the energy spectrum for many im- purities}

Let us generalize previous results for the case of $l$ randomly distributing impurity centers at the positions $k_{1}, \ldots, k_{l}$. In contrast to Anderson disorder, which randomizes the Hamiltonian matrix of the whole system, here we have ideal ones of the periodic system:

$$
\Delta_{N}=\left|\begin{array}{ccccccc}
M_{k_{1}-1}^{0} & 1 & & & & & 0 \\
1 & -x_{1} & 1 & & & & \\
& 1 & M_{k_{2}-k_{1}-1}^{0} & 1 & & & \\
& & 1 & -x_{1} & 1 & & \\
& & & 1 & \cdot & 1 & \\
0 & & & & 1 & -x_{1} & 1 \\
& & & & & 1 & M_{N-k_{l}}^{0}
\end{array}\right|=0
$$

Mathematical Modeling and Computing, Vol.5, No. 2, pp. 147-157 (2018) 
where the matrices $M^{0}$ correspond to determinant (4) of ideal sub-systems with respective sizes. To find the energy spectrum, we need to solve a secular problem of $N \times N$ size. One of the disadvantages of one-electron approximation is the numerical complexity of machine calculations of such systems. Therefore, the problem is to reduce the secular problem of size $N \times N$ to only the equation of the order $N$. In consistently conducting calculations, similar to previous, we obtain the formula:

$$
\Delta_{l+1}^{k_{l+1}-1}=\sum_{i=0}^{l} a_{l+1-i}\left(x-x_{1}\right)^{i},
$$

where $a_{l+1}=\Delta_{l+1}^{0\left(k_{l+1}-1\right)}$

$$
\begin{aligned}
& a_{l}=\sum_{i=0}^{l-1} \Delta^{0\left(k_{l+1}-k_{l-j}-1\right)} \Delta_{l-j}^{0\left(k_{l-j}-1\right)}, \\
& a_{1}=\Delta_{1}^{0\left(k_{1}-1\right)} \Delta^{0\left(k_{l+1}-k_{l}-1\right)} \ldots \Delta^{0\left(k_{2}-k_{1}-1\right)} .
\end{aligned}
$$

This formula is more convenient in recurrent representation. Let us expand the determinant by $k_{l}$ column; after some simple algebra, we have

$$
\Delta_{N}^{(l)}=\Delta_{N}^{(l-1)}+\left(x-x_{1}\right) \Delta_{N-k_{l}}^{0} \Delta_{k_{l}-1}^{(l-1)} .
$$

We succeeded in transformation the secular problem to an equation (7) of the order $N$, which can be solved numerically. Seeking energies can be found as $\varepsilon_{\mu}=E_{0}+V x, x=-2 \cos \Theta$. The results are convenient to present by constructing a configurative averaged density of states.

\subsection{Configuration averaged density of states}

By definition, the density of energy states is defined as relations $\rho(E)=\Delta N(E) / N$, where $\Delta N(E)$ is a number of energy states in $(E, E+d E)$ interval. Density of states is the main characteristic for the study of energy spectra. We construct a configuration averaged density of states for all combinations

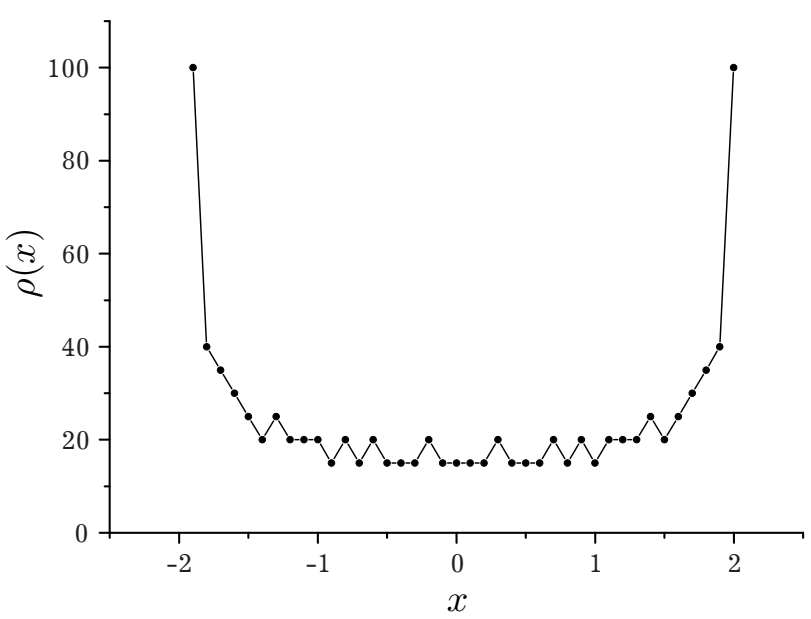

Fig. 5. Configuration averaged density of states of an ideal chain of ions $(N=100, \Delta=0)$. of impurities at $k_{1}, \ldots, k_{l}$ positions in the following form:

$$
\bar{\rho}(x)=\frac{1}{N_{\text {conf }}} \sum_{k_{1}, \ldots, k_{l}} \frac{\Delta N(x)}{\Delta x},
$$

where $k_{1}=1, \ldots, N-1, k_{2}=k_{1}+1, \ldots, N, \ldots$, $k_{l}=k_{l-1}+1, \ldots, N$,

$$
N_{\text {conf }}=\frac{N !}{l !(N-l) !}=\frac{(N-l+1) \ldots N}{l !},
$$

here $\Delta N(x)$ is a number of the roots in the interval of dimensionless energies $\Delta x$. Configuration averaged density of states of an ideal chain of ions and at the disturbance $\Delta=0.4$ are shown in Fig. 5 and Fig. 6, respectively. The spread of values is due to the finiteness of the chain.

Results for larger disturbance $\Delta$ which leads to the emergence of decoupled levels (in order to the condition $\Delta>(N+1) / N)$ are shown in Fig. 7. An impurity, such as substitutional ion or a vacancy in the porous carbon can lead to a quasi-bound state near the Fermi level. A small concentration of 


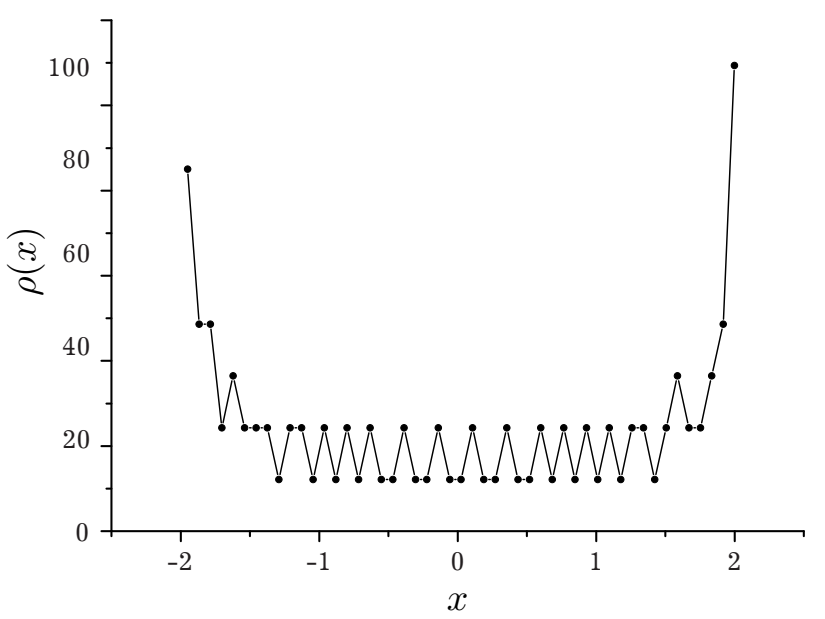

Fig. 6. Configuration averaged density of states $(N=100,2$ impurities, the disturbance $\Delta=0.4)$.

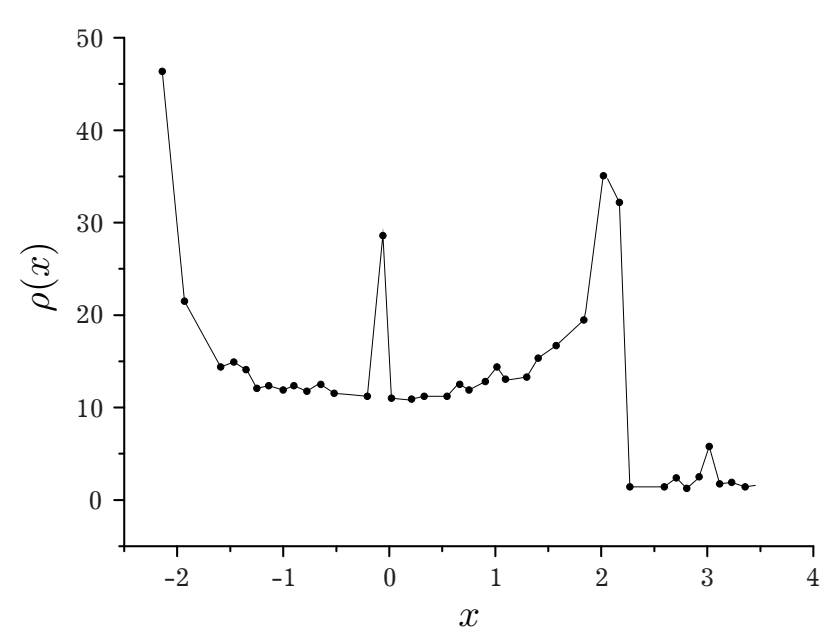

Fig. 7. Configuration averaged density of states $(N=60,2$ impurities, the disturbance $\Delta=2)$.

impurities can induce decoupled levels. As seen from Fig. 7, one new resonant state appears above the Fermi level. An impurity with negative strength has similar effects as the one with positive strength, except that the resonant state induced by the impurity is below the Fermi energy. The resonant state associated with positive or negative energy is analogous to the acceptor or donor state in semiconductors, which lead to a resonant state below or above the Fermi level, respectively. It has a great influence on conductance: the stronger the impurity, the larger the conductance.

Thus, our theoretical approaches focused on 1D lattice model of $N$ ions, confined in cylindrical subnanometer pores, are completely correspond to obtained experimental data which reveal the presence of cylindrical pores closed on one side. We have also shown that the effect of additional off-diagonal disorder of interionic interaction leads to the following consequences:

- increasing of density of states on and above the Fermi level caused by impurities (i. e. increasing of concentration of delocalized carriers) which directly leads to a decrease in the degree of releasing of Helmholtz capacitance by the capacitance of spatial space charge, thus providing as a result of the growth of the differential capacitance;

- the emergence of localized levels in order to the condition $\Delta>(N+1) / N$;

- although the Coulomb potential in free space is long-ranged, electrostatic interactions are strongly screened in a cylindrical pore, thus enables a denser packing of ions, providing correspondingly the growth of differential capacitance;

- improvement of parameters of kinetics of ions, which not only increases the growth of differential capacities, but also increases the power of the corresponding supercapacitors.

Extensions of this work may include the analysis of differential capacitance of systems in which larger clusters of impurities may occur. These phenomena could be analyzed using the same matrix techniques as employed here.

\section{Conclusion}

In this paper, we have focused on both experimental and theoretical approaches for studying the processes of charge storage and the electronic structure of synthesized sub-nanometer porous carbon. The achieved high values of the differential capacitance can be explained within the constructed impedance model by presence of sub-nanometer cylindrical pores when essentially only one row of ions can fit into the pore. $1 \mathrm{D}$ lattice model where the interactions between ions take place via nearest neighbors has given us an excellent opportunity to analyze charging processes analytically. It was presented a recursion formula method to compute the energy spectrum for a local defect and for the $l$ randomly 
distributing impurity centers at the positions $k_{1}, \ldots, k_{l}$. There is a role of the additional off-diagonal disorder, an effect of the additional hopping parameter in the energy spectrum is included in the analytic result given in the equation (6). We reduce the secular problem of the $N \times N$ size to the single equation (7) of the order of $N$. The main characteristic of the electron spectrum - the density of states is constructed to study the mechanism of the charge storage, its dependence on the interaction between ions, the effect of impurities on energy spectrum.

[1] Gryglewicz G., Machnikowski J., Lorenc-Grabowska E., Lota G., Frackowiak E. Effect of pore size distribution of coal-based activated carbons on double layer capacitance. Electrochimica Acta. 50 (5), 1197-1206 (2005).

[2] Conway B. Electrochemical Supercapacitors. New York, Plenum Publishing (1999).

[3] Abioye A. M., Ani F. N. Recent development in the production of activated carbon electrodes from agricultural waste biomass for supercapacitors: A review. Renewable and Sustainable Energy Reviews. 52, 1282-1293 (2015).

[4] David B. Optimization of power and energy densities in supercapacitors. Journal of Power Sources. 195, 748-3756 (2010).

[5] Gonzalez A., Goikolea E., Barrena J. A., Mysyk R. Review on supercapacitors: Technologies and materials. Renewable and Sustainable Energy Reviews. 58, 1189-2006 (2016).

[6] Zhan C., Lian C., Zhang Yu, Thompson M.W., Xie Yu, Wu J., Kent P. R. C., Cummings P. T., Jiang D., Wesolowski D. J. Computational insights into materials and interfaces for capacitive energy storage. Adv. Sci. 4 (7), 1700059 (2017).

[7] Salanne M., Rotenberg B., Naoi K., Kaneko K., Taberna P.-L., Grey C. P., Dunn B., Simon P. Efficient storage mechanisms for building better supercapacitors. Nature Energy. 1, 1-10 (2016).

[8] Chmiola J., Yushin G., Gogotsi Y., Portet C., Simon P., Taberna P. L. Anomalous Increase in Carbon Capacitance at Pore Sizes Less Than 1 Nanometer. Science. 313, 1760-1763 (2006).

[9] Len J. Supramolecular chemistry. Concepts and perspectives. Novosybirsk, Nauka (1998), (in Russian).

[10] Stid D., Etvud D. Supramolecular chemisty. Moscow, Akademkniga (2007), (in Russian).

[11] Stojnov S. B., Grafov B. M., Savova-Stojnova B., Yelkin V. V. Electrochemical impedance. Moscow, Nauka (1991), (in Russian).

[12] Barsoukov E., Macdonald J. Impedance spectroscopy. Theory, experiment and application. Wiley R. Interscience, Canada (2005).

[13] Kondrat S., Kornyshev A. Superionic state in double-layer capacitors with nanoporous electrodes. Journal of Physics: Condensed Matter. 23, 2-10 (2010).

[14] Skinner B., Chen T., Loth M. S., Shklovskii B.I. Theory of volumetric capacitance of an electric double-layer supercapacitor. Phys. Rev. E. 83, 056102 (2011).

[15] Merlet A., Péan C., Rotenberg B., Madden P. A., Daffos B., Taberna P.-L., Simon P., Salanne M. Highly confined ions store charge more efficiently in supercapacitors. Nature Communications. 4, 2701 (2013).

[16] Lee A. A., Kondrat S., Kornyshev A. A. Single-File Charge storage in conducting nanopores. Phys. Rev. Lett. 113, 048701 (2014).

[17] Rochester C., Sartor A., Pruessner G., Kornyshev A. A. "One dimensional" double layer. The effect of size asymmetry of cations and anions on charge-storage in ultranarrownanopores - an Ising model theory. Russian Journal of Electrochemistry. 53 (10), 1165-1170 (2017).

[18] Hu G. Y., O'Connell R. F. Analytical inversion of symmetric tridiagonal matrices. J. Phys: A.: Math. Gen. 29, 1511-1513 (1996). 


\title{
Вплив додаткового позадіагонального безладу міжіонної взаємодії на зберігання заряду у субнанопорах супрамолекулярного карбону суперконденсаторів
}

\author{
Клапчук М., Григорчак I. \\ Національний університет "Лъвівсъка політехніка", \\ вул. С. Бандери, 12, 79013, Лъвів, Україна
}

В роботі методами супрамолекулярної інженерії вперше синтезовано субнанопористий карбон для ефективного накопичення енергії на межі його розділу з електролітом. Досягнуте значення диференціальної ємності більш як вдвічі перевищуе відповідну величину для відомих карбонових структур. На основі побудованої імпедансної моделі та мікроскопічної теорії запропоновано фізичні механізми досліджених процесів.

Ключові слова: супрамолекулярні ансамблі, нанопористий карбон, пориста структура, імпедансна спектроскопія, діаграма Найквіста, 1 м модель подвійного електричного шару, метод рівнянь руху.

2000 MSC: 82D15, 82D20

УДК: 541.136 .2 